\title{
VIBRATION OF A COMPOSITE ELASTIC BEAM ON AN INHOMOGENEOUS ELASTIC FOUNDATION
}

\author{
Onur Şahin \\ Faculty of Arts and Sciences, Department of Mathematics, Giresun University, Giresun, Turkey \\ onur.sahin@giresun.edu.tr
}

Received: 3 March 2020; Accepted: 8 June 2020

\begin{abstract}
The lowest vibration modes of a composite beam resting on an inhomogeneous elastic foundation in the case of a clamped-free boundary condition are investigated. It is observed that the relation between the frequency and foundation constant has an effect on the valid solution of the problem. An asymptotic procedure is employed to derive the eigenfrequencies as well as the eigenforms revealing that only under certain conditions on the ratios of material parameters. Numerical illustrations are presented to confirm that the obtained asymptotic frequencies agree well with the exact frequencies in the lowest frequency range. Comparisons of asymptotic and exact displacements are also presented and a remarkable agreement is observed for high-contrast beam components.
\end{abstract}

MSC 2010: 74A40, 74H45, $74 G 10$

Keywords: composite beam, elastic foundation, low-frequency vibration, perturbation method

\section{Introduction}

The vibration of composite elastic materials has been extensively studied by many researchers since it appears in numerous applications in industries, including photovoltaic panels [1], energy harvesting devices [2], laminated glass beams [3], etc. With the latest technological developments, materials with high contrast properties have become an important application area in metamaterial designs [4] and soft robotics [5-7]. We also cite [8] investigating Rayleigh type wave dispersion in inhomogeneous media. The low-frequency vibrations in multi-layered structures are also another research topic that has attracted much attention recently. The lowest longitudinal vibrations of piecewise inhomogeneous string [9], rod [10,11] and beam [12,13] were studied. In [10], an asymptotic model leading to the estimation of low natural frequencies and the displacement profile was developed. This model was then generalized to beam in [13]. Thus, it has been presented that even though the rod has one rigid body motion, the beam has two rigid body motions including rotation and translation. 
The vibration of a beam resting on an elastic foundation which is generally used in the investigation of the foundations of buildings, highway and railroad structures is another research area and has been tread by numerous authors, see [14,16]. Although there are many models for elastic foundation, the Winkler model is the most adopted one, in which the response of a normally applied foundation is proportional at every point to deflection of the beam. A general method for the vibration analysis of beams on variable Winkler elastic foundations has been presented in [17]. Doyle [18] investigated the natural response of a partially supported elastic beam when both ends are either simply supported or free. Beams having different cross-sectional areas on elastic foundation are analyzed in [19]. This analysis provided a simple procedure to evaluate the effect of elastic foundations on the free vibration of stepped beams. Non-linear vibrations of an elastic beam resting on an elastic foundation were also investigated in $[20,22]$. Free vibrations of micro and nano beams resting on an elastic foundation are other research areas that have been studied intensively in recent years, e.g. see [23] and [24] and references therein. We also mention [25] which generalizes Eringen's result for a random response of a beam on elastic foundation.

This paper is devoted to the analysis of low-frequency vibrations of a strongly piecewise inhomogeneous beam resting on an elastic foundation consisting of two different parts. The beam consists of two components having different material and geometrical properties. The analysis shows that the valid solution of the problem depends on the relation between the frequency and elastic foundation constant. An asymptotic model in which certain restrictions are imposed on the material properties such as Young moduli, densities and lengths is employed to obtain approximate eigenfrequencies and displacements [10]. It is also shown that the composite beam resting on an elastic foundation has two rigid body motions like a strongly inhomogeneous beam without an elastic foundation [12].

This paper is organized as follows: In Section 2, the governing equation with continuity and end conditions is presented. Then, for further reference, the exact solutions of a two-component beam resting on two different elastic foundations with clamped-free ends are given for two different cases of dimensionless constant corresponding difference of non-dimensional frequency and elastic foundation constant. In Section 3, the perturbation analysis is established through the small parameter defined by the high contrast of material properties. The necessary restrictions on the material parameters are determined in order to get a low-frequency regime. The established asymptotic model is employed to the beam with two different cases which results in approximate eigenfrequencies and displacements. Section 4 contains some numerical comparisons of the exact and asymptotic results for eigenfrequencies and eigenforms. 


\section{Statement of the problem}

Consider time-harmonic vibrations of a finite rectangular inhomogeneous beam with two components composed of alternating soft and stiff parts of arbitrary lengths, which rest on an elastic foundation consisting of two different partse (Fig. 1). The governing equations for each component of the beam are given by

$$
\frac{d^{4} y_{i}}{d x^{4}}-\frac{\omega^{2}}{a_{i}^{2}} y_{i}+\frac{k_{i}}{D_{i}} y_{i}=0, \quad i=1,2,
$$

where $y_{i}$ are the displacements, $x$ is the longitudinal coordinates, $\omega$ is the angular frequency, $a_{i}=\sqrt{D_{i} / M_{i}}, k_{i}$ are the moduli of elastic foundation, $D_{i}=E_{i} I$ are the stiffness rigidities and $M_{i}=\rho_{i} A$ are linear mass densities with $E_{i}, I, \rho_{i}$ and $A$ denoting Young's moduli, moment of inertia, material densities and cross-sectional area, respectively.

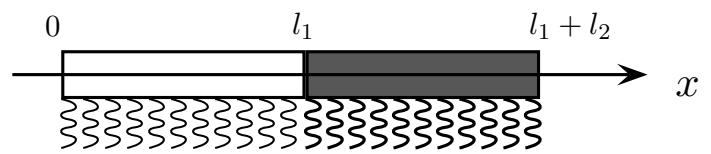

$k_{1}$

$k_{2}$

Fig. 1. A composite beam resting on an inhomogeneous elastic foundation

Introducing non-dimensional local longitudinal coordinates by

$$
\xi_{i}=\frac{x}{l_{i}}, \quad i=1,2,
$$

equation (1) may be rewritten as

$$
\frac{d^{4} y_{i}}{d \xi_{i}^{4}}-\alpha_{i}^{4} y_{i}=0, \quad i=1,2,
$$

where

$$
\alpha_{i}^{4}=\lambda_{i}^{4}-\beta_{i}^{4}
$$

and

$$
\lambda_{i}^{4}=\frac{l_{i}^{4} \omega^{2}}{a_{i}^{2}}, \quad \beta_{i}^{4}=\frac{l_{i}^{4} k_{i}}{D_{i}} .
$$

In what follows, we will restrict our analysis to a beam with clamped-free ends for which the outer edges satisfy

$$
\begin{array}{r}
y_{1}(0)=y_{1}^{\prime}(0)=0, \\
y_{2}^{\prime \prime}(1+l)=y_{2}^{\prime \prime \prime}(1+l)=0,
\end{array}
$$

where $l=l_{1} / l_{2}$. 
The displacements, stresses, bending moments and shear forces of the beam must be continuous at the interface which are given, respectively, by

$$
\begin{array}{ll}
y_{1}(1)=y_{2}(l), & y_{1}^{\prime}(1)=l y_{2}^{\prime}(l), \\
D y_{1}^{\prime \prime}(1)=l^{2} y_{2}^{\prime \prime}(l), & D y_{1}^{\prime \prime \prime}(1)=l^{3} y_{2}^{\prime \prime \prime}(l),
\end{array}
$$

where $D=D_{1} / D_{2}$. We also introduce non-dimensional quantities

$$
M=\frac{M_{1}}{M_{2}}, \quad a=\frac{a_{1}}{a_{2}} .
$$

It can be easily seen from equation (4) that even if the non-dimensional frequencies, $\lambda_{i}$, and foundation constants, $\beta_{i}$, are always positive, $\alpha_{i}^{4}$ could be positive, negative or zero. Therefore, the solution of the formulated problem depends on the sign of $\alpha_{i}^{4}$

In the case $\alpha_{i}^{4}>0$, the solution of equation (3) is written as

$$
y_{i}\left(\xi_{i}\right)=A_{i} \cos \left(\alpha_{i} \xi_{i}\right)+B_{i} \sin \left(\alpha_{i} \xi_{i}\right)+C_{i} \cosh \left(\alpha_{i} \xi_{i}\right)+D_{i} \sinh \left(\alpha_{i} \xi_{i}\right) .
$$

When $\alpha_{i}^{4}=0$ the corresponding beam displacement has a cubic polynomial form

$$
y_{i}\left(\xi_{i}\right)=A_{i} \xi_{i}^{3}+B_{i} \xi_{i}^{2}+C_{i} \xi_{i}+D_{i}
$$

while the case $\alpha_{i}^{4}<0$ results in the following solution

$$
\begin{aligned}
y_{i}\left(\xi_{i}\right)= & A_{i} \cos \left(\frac{\alpha_{i} \xi_{i}}{\sqrt{2}}\right) \cosh \left(\frac{\alpha_{i} \xi_{i}}{\sqrt{2}}\right)+B_{i} \cos \left(\frac{\alpha_{i} \xi_{i}}{\sqrt{2}}\right) \sinh \left(\frac{\alpha_{i} \xi_{i}}{\sqrt{2}}\right) \\
& +C_{i} \sin \left(\frac{\alpha_{i} \xi_{i}}{\sqrt{2}}\right) \cosh \left(\frac{\alpha_{i} \xi_{i}}{\sqrt{2}}\right)+D_{i} \sin \left(\frac{\alpha_{i} \xi_{i}}{\sqrt{2}}\right) \sinh \left(\frac{\alpha_{i} \xi_{i}}{\sqrt{2}}\right) .
\end{aligned}
$$

It is obvious that there are nine different combinations of signs of $\alpha_{1}^{4}$ and $\alpha_{2}^{4}$ in which only a few of them cause a proper solution. For example, if $\alpha_{1}^{4}>0$ and $\alpha_{2}^{4}>0$ the problem becomes identical to a composite beam without an elastic foundation which has been extensively analyzed in [12]. However, $\alpha_{1}^{4}=0, \alpha_{2}^{4}=0$ and $\alpha_{1}^{4}<0, \alpha_{2}^{4}<0$ do not constitute a valid solution for the considered beam.

In this paper, apart from the aforementioned cases, we will only consider two combinations of $\alpha_{1}^{4}$ and $\alpha_{2}^{4}$ which are $\alpha_{1}^{4}=0, \alpha_{2}^{4}>0$ and $\alpha_{1}^{4}>0, \alpha_{2}^{4}<0$. The rest of them can be analyzed in a similar manner.

(i) $\alpha_{1}^{4}=0$ and $\alpha_{2}^{4}>0$ :

In the present case, the displacements corresponding to the first and second components of the beam are written as

$$
\begin{aligned}
& y_{1}\left(\xi_{1}\right)=A_{1} \xi_{1}^{3}+B_{1} \xi_{1}^{2}+C_{1} \xi_{1}+D_{1}, \\
& y_{2}\left(\xi_{2}\right)=A_{2} \cos \left(\alpha_{2} \xi_{2}\right)+B_{2} \sin \left(\alpha_{2} \xi_{2}\right)+C_{2} \cosh \left(\alpha_{2} \xi_{2}\right)+D_{2} \sinh \left(\alpha_{2} \xi_{2}\right) .
\end{aligned}
$$


Applying boundary conditions (6) and continuity conditions (7) to (10) yields eight homogeneous equations of the form

$$
\left[\mathbb{M}_{1}\right][A]=0
$$

where $[A]=\left(A_{1}, B_{1}, C_{1}, D_{1}, A_{2}, B_{2}, C_{2}, D_{2}\right)$ and $\left[\mathbb{M}_{1}\right]$ is the matrix of coefficients given in the Appendix. This set of linear equations has a non-trivial solution provided that

$$
\left|\mathbb{M}_{1}\right|=0
$$

resulting in the following sought frequency equation

$$
\begin{aligned}
12 D^{2}+l^{4} \alpha_{2}^{4} & +\left(\left(12 D^{2}-l^{4} \alpha_{2}^{4}\right) \cos \alpha_{2}-4 D l \alpha_{2}\left(l^{2} \alpha_{2}^{2}+3\right) \sin \alpha_{2}\right) \cosh \alpha_{2} \\
& -4 D l \alpha_{2}\left(\left(l^{2} \alpha_{2}^{2}-3\right) \cos \alpha_{2}+3 l \alpha_{2} \sin \alpha_{2}\right) \sinh \alpha_{2}=0 .
\end{aligned}
$$

(ii) $\alpha_{1}^{4}>0$ and $\alpha_{2}^{4}<0$ :

The displacements for the components of the beam in the considered case are written as

$$
\begin{aligned}
y_{1}\left(\xi_{1}\right) & =A_{1} \cos \left(\alpha_{1} \xi_{1}\right)+B_{1} \sin \left(\alpha_{1} \xi_{1}\right)+C_{1} \cosh \left(\alpha_{1} \xi_{1}\right)+D_{1} \sinh \left(\alpha_{1} \xi_{1}\right), \\
y_{2}\left(\xi_{2}\right) & =A_{2} \cos \left(\frac{\alpha_{2} \xi_{2}}{\sqrt{2}}\right) \cosh \left(\frac{\alpha_{2} \xi_{2}}{\sqrt{2}}\right)+B_{2} \cos \left(\frac{\alpha_{i} \xi_{2}}{\sqrt{2}}\right) \sinh \left(\frac{\alpha_{2} \xi_{2}}{\sqrt{2}}\right) \\
& +C_{2} \sin \left(\frac{\alpha_{2} \xi_{2}}{\sqrt{2}}\right) \cosh \left(\frac{\alpha_{2} \xi_{2}}{\sqrt{2}}\right)+D_{2} \sin \left(\frac{\alpha_{2} \xi_{2}}{\sqrt{2}}\right) \sinh \left(\frac{\alpha_{2} \xi_{2}}{\sqrt{2}}\right) .
\end{aligned}
$$

Substituting equations in (16) into equations (6)-(7) gives us again a linear system of equations in $\left(A_{1}, B_{1}, C_{1}, D_{1}, A_{2}, B_{2}, C_{2}, D_{2}\right)$ with a coefficient matrix $\left[\mathbb{M}_{2}\right]$ given in the Appendix. Similarly to the previous case, the solvability of this linear algebraic system leads to the frequency equation given by

$$
\begin{aligned}
& l^{4} \alpha_{1}^{4}\left(\cos \alpha_{1} \cosh \alpha_{1}-1\right) \cos \left(-\left(\sqrt{ } 2 \alpha_{2}\right)+\cosh (\sqrt{ } 2 \alpha z)-D\right)^{2} \alpha_{1}^{4}\left(\cos \alpha_{1} \cosh \alpha_{1}+1\right) \\
& \times\left(\cos \left(\sqrt{2} \alpha_{2}\right)+\cosh \left(\sqrt{2} \alpha_{2}\right)+2\right)+2 D l^{2} \alpha_{1}^{2} \alpha_{2}^{2}\left(\cos \left(\sqrt{2} \alpha_{2}\right)-\cosh \left(\sqrt{2} \alpha_{2}\right)\right) \sin \alpha_{1} \sinh \alpha_{1} \\
& +\sqrt{2} D l \alpha_{1}^{3} \alpha_{2}\left(\cosh \alpha_{1} \sin \alpha_{1}+\cos \alpha_{1} \sinh \alpha_{1}\right)\left(\sin \left(\sqrt{2} \alpha_{2}\right)-\sinh \left(\sqrt{2} \alpha_{2}\right)\right) \\
& +\sqrt{2} D l^{2} \alpha_{1} \alpha_{2}^{3}\left(\cosh \alpha_{1} \sin \alpha_{1}-\cos \alpha_{1} \sinh \alpha_{1}\right)\left(\sin \left(\sqrt{2} \alpha_{2}\right)+\sinh \left(\sqrt{2} \alpha_{2}\right)\right)=0 .
\end{aligned}
$$

\section{Asymptotic analysis}

The low-frequency vibrations of multi-component beams have received an increased amount of attention after the development of new materials including soft robotics $[6,7,11]$. Therefore, in this section, we study the lowest eigenmodes of the beams shown in Figure 1. As can be seen from the previous section, determin- 
ing the exact eigenfrequencies and corresponding exact eigenmodes for the considered problem is substantially difficult. Therefore, we use asymptotic techniques earlier developed for longitudinal vibrations of strongly inhomogeneous elastic rods and beams $[10,12]$, which make the analysis of the frequencies and displacements easier. In this context, we first assume that Young's modulus of the stiffer component is much greater than the softer one, i.e.

$$
\varepsilon=\frac{D_{1}}{D_{2}} \ll 1
$$

is a small parameter signifying the high contrast material properties.

Let us now expand the frequency parameters and displacements into the asymptotic series

$$
\begin{aligned}
& \lambda_{i}^{4}=\varepsilon\left(\lambda_{i, 0}^{4}+\varepsilon \lambda_{i, 1}^{4}+\varepsilon^{2} \lambda_{i, 2}^{4}+\cdots\right), \\
& y_{i}=y_{i, 0}+\varepsilon y_{i, 1}+\varepsilon^{2} y_{i, 2}+\cdots, \quad i=1,2
\end{aligned}
$$

which may also correspond to the low frequency regimes of the problems mentioned at the beginning of this section. We also assume that the scaled foundation constant is order of $\varepsilon$, i.e.

$$
\beta_{i}^{4}=\varepsilon \beta_{i, 0}^{4}
$$

Therefore $\alpha_{i}^{4}$ may be written as asymptotic series, yielding

$$
\alpha_{i}^{4}=\varepsilon\left(\alpha_{i, 0}^{4}+\varepsilon \alpha_{i, 1}^{4}+\varepsilon^{2} \alpha_{i, 2}^{4}+\cdots\right)
$$

where $\alpha_{i, 0}^{4}=\lambda_{i, 0}^{4}-\beta_{i, 0}^{4}$ and $\alpha_{i, j}^{4}=\lambda_{i, j}^{4}, i=1,2$ and $j=1,2, \ldots$. Keeping in mind that $\alpha_{1}^{4} \sim \alpha_{2}^{4} \sim \varepsilon$ and $\beta_{1}^{4} \sim \beta_{2}^{4} \sim \varepsilon$ over the low-frequency range of interest, we should have the following asymptotic order relations;

$$
\frac{M_{1}}{M_{2}} \sim \varepsilon, \quad l \sim 1, \quad a \sim 1 \quad k=\frac{k_{1} / D_{1}}{k_{2} / D_{2}} \sim 1 .
$$

It may be readily observed from (19)-(21) that $\alpha_{1}^{4}$ and $\alpha_{2}^{4}$ are related to each other by

$$
\alpha_{1}^{4}=\frac{l^{4}}{a^{2}}\left(\alpha_{2}^{4}+\beta_{2}^{4}\left(1-k a^{2}\right)\right) .
$$

Therefore, the sign of $\alpha_{i}^{4}$ depending on the value of $k a^{2}$ can be given in Table 1 .

Table 1. Sign of $\alpha_{i}^{4}$ with respect to $k a^{2}$

\begin{tabular}{|c|c|c|}
\cline { 2 - 3 } \multicolumn{1}{c|}{} & $k a^{2}>1$ & $k a^{2}<1$ \\
\hline$\alpha_{1}^{4}>0$ & $\alpha_{2}^{4}>0$ & $\alpha_{2}^{4}>0$ or $\alpha_{2}^{4}<0$ or $\alpha_{2}^{4}=0$ \\
\hline$\alpha_{1}^{4}=0$ & $\alpha_{2}^{4}>0$ & $\alpha_{2}^{4}<0$ \\
\hline$\alpha_{1}^{4}<0$ & $\alpha_{2}^{4}>0$ or $\alpha_{2}^{4}<0$ or $\alpha_{2}^{4}=0$ & $\alpha_{2}^{4}<0$ \\
\hline
\end{tabular}


Bearing in mind the definition of the small parameter, the continuity conditions at the interface and the boundary conditions take the form

$$
\begin{array}{ll}
y_{1}(1)=y_{2}(l), & y_{1}^{\prime}(1)=l y_{2}^{\prime}(l), \\
\varepsilon y_{1}^{\prime \prime}(1)=l^{2} y_{2}^{\prime \prime}(l), & \varepsilon y_{1}^{\prime \prime \prime}(1)=l^{3} y_{2}^{\prime \prime \prime}(l),
\end{array}
$$

and

$$
\begin{aligned}
& y_{1}(0)=y_{1}^{\prime}(0)=0, \\
& y_{2}^{\prime \prime}(1+l)=y_{2}^{\prime \prime \prime}(1+l)=0,
\end{aligned}
$$

respectively.

On substituting the asymptotic expansions (19) into (3), (24) and (25), we have the boundary value problem given by

$$
\frac{d^{4} y_{i, 0}}{d \xi_{i}^{4}}+\varepsilon\left(\frac{d^{4} y_{i, 1}}{d \xi_{i}^{4}}-\alpha_{i}^{4} y_{i, 0}\right)+\cdots=0
$$

with

$$
\begin{aligned}
& y_{2,0}(l)-y_{1,0}(1)+\varepsilon\left(y_{2,1}(l)-y_{1,0}(1)\right)+\cdots=0, \\
& l y_{2,0}^{\prime}(l)-y_{1,0}^{\prime}(1)+\varepsilon\left(l y_{2,1}^{\prime}(l)-y_{1,0}^{\prime}(1)\right)+\cdots=0, \\
& l^{2} y_{2,0}^{\prime \prime}(l)+\varepsilon\left(l^{2} y_{2,1}^{\prime \prime}(l)-y_{1,0}^{\prime \prime}(1)\right)+\cdots=0, \\
& l^{3} y_{2,0}^{\prime \prime \prime}(l)+\varepsilon\left(l^{3} y_{2,1}^{\prime \prime \prime}(l)-y_{1,0}^{\prime \prime \prime}(1)\right)+\cdots=0,
\end{aligned}
$$

and

$$
\begin{aligned}
& y_{1,0}(0)+\varepsilon y_{1,1}(0)+\cdots=y_{1,0}^{\prime}(0)+\varepsilon y_{1,1}^{\prime}(0)+\cdots=0, \\
& y_{2,0}^{\prime \prime}(0)+\varepsilon y_{2,1}^{\prime \prime}(0)+\cdots=y_{2,0}^{\prime \prime \prime}(0)+\varepsilon y_{2,1}^{\prime \prime \prime}(0)+\cdots=0 .
\end{aligned}
$$

Thus, regardless of the sign of $\alpha_{i}^{4}$, at the leading order the equations of motion become

$$
\frac{d^{4} y_{i, 0}}{d \xi_{i}^{4}}=0
$$

subject to the continuity

$$
\begin{aligned}
& y_{1,0}(1)=y_{2,0}(l), \quad y_{1,0}^{\prime}(1)=l y_{2,0}^{\prime}(l), \\
& y_{2,0}^{\prime \prime}(l)=y_{2,0}^{\prime \prime \prime}(l)=0
\end{aligned}
$$

and the boundary conditions

$$
\begin{aligned}
& y_{1,0}(0)=y_{1,0}^{\prime}(0)=0, \\
& y_{2,0}^{\prime \prime}(1+l)=y_{2,0}^{\prime \prime \prime}(1+l)=0 .
\end{aligned}
$$


The solution of the boundary value problem (29)-(31) may be written as

$$
\begin{aligned}
& y_{1,0}=A_{1} \xi_{1}^{3}+B_{1} \xi_{1}^{2}, \\
& y_{2,0}=A_{2} \xi_{2}+B_{2},
\end{aligned}
$$

which correspond to leading order displacements for the soft and stiff parts of the beam respectively. The coefficients of the soft and stiff displacements are also related to each other from equation $\left(30_{1}\right)$ and $\left(30_{2}\right)$ by

$$
A_{1}=-2 B_{2}-l A_{2}, \quad B_{1}=3 B_{2}+2 l A_{2} .
$$

We now proceed to the next order problem for the stiff component of the beam, which is formulated from (26)-(28) as

$$
\frac{d^{4} y_{2,1}}{d \xi_{2}^{4}}-\alpha_{2,0}^{4} y_{2,0}=0
$$

with continuity

$$
\left.\frac{d^{2} y_{2,1}}{d \xi_{2}^{2}}\right|_{\xi_{2}=l}=\left.\frac{1}{l^{2}} \frac{d^{2} y_{1,0}}{d \xi_{1}^{2}}\right|_{\xi_{1}=1},\left.\quad \frac{d^{3} y_{2,1}}{d \xi_{2}^{3}}\right|_{\xi_{2}=l}=\left.\frac{1}{l^{3}} \frac{d^{3} y_{1,0}}{d \xi_{1}^{3}}\right|_{\xi_{1}=1},
$$

and boundary conditions

$$
\left.\frac{d^{2} y_{2,1}}{d \xi_{2}^{2}}\right|_{\xi_{2}=1+l}=\left.\frac{d^{3} y_{2,1}}{d \xi_{2}^{3}}\right|_{\xi_{2}=1+l}=0 .
$$

(i) $\alpha_{1}^{4}=0$ and $\alpha_{2}^{4}>0$ :

If $\alpha_{2}^{4}>0$, the leading order term of expansion for $\alpha_{2}^{4}$ becomes greater than zero, namely $\alpha_{2,0}^{4}>0$. First substituting expression of $y_{1,0},\left(32_{1}\right)$, into (34) and then integrating over $l \leq \xi_{2} \leq 1+l$, we obtain

$$
12 A_{1}-\alpha_{2,0}^{4} l^{3}\left((1+2 l) A_{2}+2 B_{2}\right)=0 .
$$

Next, multiplying (34) by $\xi_{2}$ and integrating over $l \leq \xi_{2} \leq 1+l$ results in

$$
12 B_{1}-\alpha_{2,0}^{4} l^{2}\left(2\left(3 l^{2}+3 l+1\right) A_{2}+3(2 l+1) B_{2}\right)=0 .
$$

The coefficients $A_{1}$ and $B_{1}$ in equations (37) and (38) can be expressed through the coefficients in (33), leading to the linear set of equations

$$
\begin{aligned}
& 12\left(l A_{2}+2 B_{2}\right)-l^{3}\left((2 l+1) A_{2}+2 B_{2}\right) \alpha_{2,0}^{4}=0, \\
& \left.24 l A_{2}+36 B_{2}-l^{2} 2\left(3 l^{2}+3 l+1\right) A_{2}+3(2 l+1) B_{2}\right) \alpha_{2,0}^{4}=0 .
\end{aligned}
$$

The latter has a non-trivial solution provided that

$$
l^{4} \alpha_{2,0}^{8}-24 l(2+l(3+2 l)) \alpha_{2,0}^{4}+144=0 .
$$


The obtained frequency equation has two non-zero solutions given by

$$
\begin{aligned}
& \alpha_{2,0}^{4}=\frac{12\left(-2(1+l) \sqrt{1+l+l^{2}}+2+l(3+2 l)\right)}{l^{3}}, \\
& \alpha_{2,0}^{4}=\frac{12\left(2(1+l) \sqrt{1+l+l^{2}}+2+l(3+2 l)\right)}{l^{3}} .
\end{aligned}
$$

(ii) $\alpha_{1}^{4}>0$ and $\alpha_{2}^{4}<0$ :

In the case $\alpha_{2}^{4}<0$, the first term of the corresponding asymptotic expansion becomes less than zero, i.e. $\alpha_{2,0}^{4}<0$. Taking into account the sign of $\alpha_{2,0}^{4}$ in equation (34) and following a similar procedure performed in the previous case, we obtain

$$
l^{4} \alpha_{2,0}^{8}+24 l(2+l(3+2 l)) \alpha_{2,0}^{4}+144=0,
$$

which is the sought frequency equation for the considered case. Thus, we arrive at two negative roots for $\alpha_{2,0}^{4}$ given by

$$
\begin{aligned}
& \alpha_{2,0}^{4}=-\frac{12\left(2(1+l) \sqrt{1+l+l^{2}}+2+l(3+2 l)\right)}{l^{3}}, \\
& \alpha_{2,0}^{4}=\frac{12\left(2(1+l) \sqrt{1+l+l^{2}}-(2+l(3+2 l))\right.}{l^{3}} .
\end{aligned}
$$

\section{Numerical results}

In this section, some numerical comparisons of exact and asymptotic formulae for the eigenfrequencies and displacements are presented. All the numerical computations are demonstrated in the case of global frequency regime for which we have $M_{1} / M_{2} \sim \varepsilon, l \sim 1, a \sim 1$ and $k \sim 1$.

Figure 2 illustrates exact, (15), and approximate, (40), frequency curves in the case $\alpha_{1}=0$ and $\alpha_{2}>0$, at $l=0.99$ and $a=1.01$. It can be easily observed from this figure that approximate frequency gives a very good approach to the exact frequency even for significant in size numerical value of $\varepsilon$. 


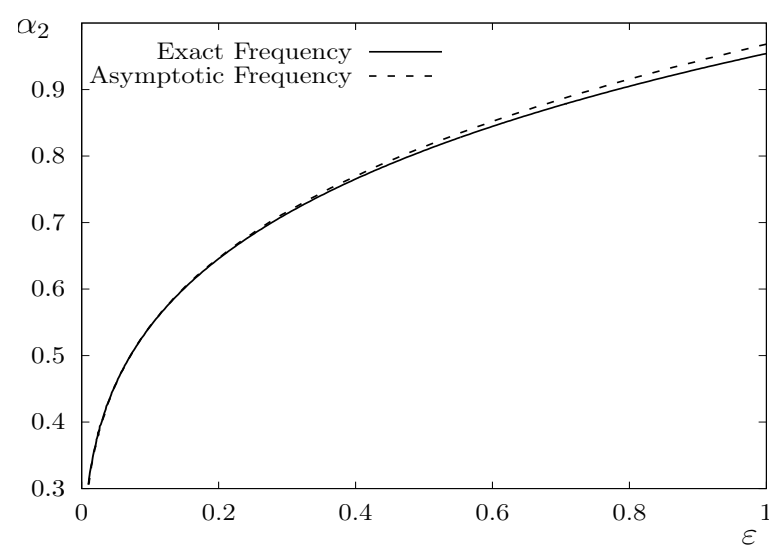

Fig. 2. Comparison of exact (15) and asymptotic (40) frequency curves at $l=0.99$ and $a=1.01$

Figure 3 shows the comparison of scaled exact and approximate displacements corresponding to eigenfrequency $\left(41_{1}\right)$ for $\alpha_{1}^{4}=0$ and $\alpha_{2}^{4}>0$. It can be readily seen from this figure that the approximate displacement (32) is, again, in quite good agreement with the exact one (12) for $\varepsilon=0.5$.

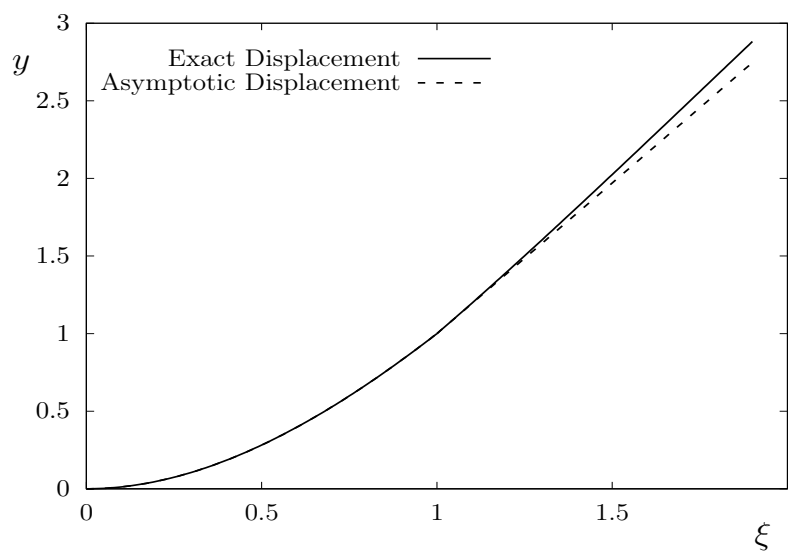

Fig. 3. Comparison of exact (12) and asymptotic (32) displacements in the case $\alpha_{1}^{4}=0$ and $\alpha_{2}^{4}>0$ for $l=0.99$ and $\varepsilon=0.5$

In the case $\alpha_{1}^{4}>0$ and $\alpha_{2}^{4}<0$, the numerical comparison of scaled exact and approximate displacements is depicted for the eigenfrequency (431) in Figure 4. In the comparison, we set $a=0.99$ and $l=0.99$ to have $\alpha_{1}^{4}>0$ and $\alpha_{2}^{4}<0$ (Table 1). Similar results can be followed for this figure. 


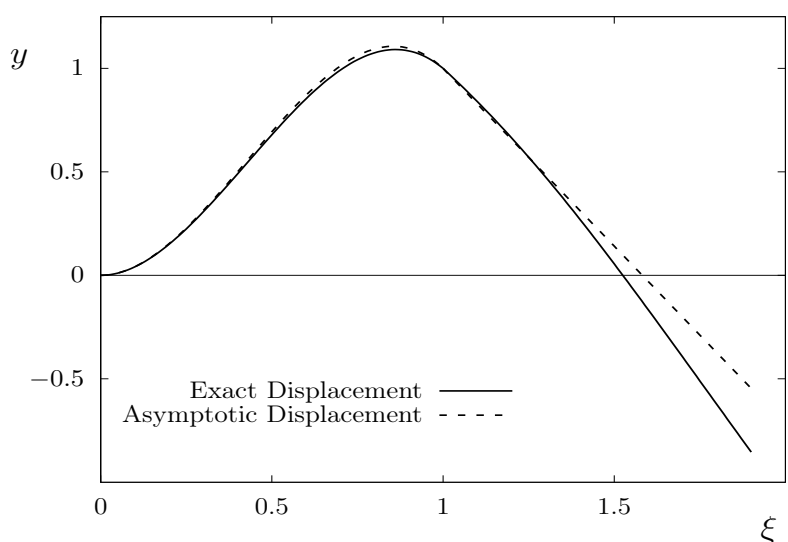

Fig. 4. Comparison of exact (16) and asymptotic (32) displacements in the case $\alpha_{1}^{4}>0$ and $\alpha_{2}^{4}<0$ for

$$
\varepsilon=0.1
$$

\section{Concluding remarks}

The low-frequency vibrations of a two-component, strongly inhomogeneous beam resting on elastic foundation have been analyzed for the clamped-free boundary condition. It is seen that the valid solution of the problem depends on the relation between the frequency and foundation constant, and only for a few cases we have a non-trivial eigenfrequency and displacement. Since the exact formulation of eigenfrequencies and eigenforms of such problems are generally given by sophisticated transcendental equations, a perturbation procedure is derived to investigate near-zero frequencies and corresponding displacements. It is also emphasized that the derived asymptotic formulae are valid for certain conditions on the ratios of material properties of the beam and foundation constants, which allow the low-frequency vibrations to be possible. Another point to note is that eigenfrequencies and eigenmodes might have been calculated through a numerical procedure (such as the FE method) however, the computational difficulty as well as the computing time, particularly when the number of the components of the beam increase, become formidable. Furthermore, applying a numerical procedure may give rise a risk to miss low-frequency eigenforms.

The proposed perturbation approach may be generalized to multi-parametric problems such as n-component strongly inhomogeneous beams and multi-layered plates. Different kinds of elastic foundation models may also be studied. The effect of the boundary conditions on the solvability of such problems is another problem that can be considered. 


\section{Appendix}

The coefficient matrixes that appear in Section 2 are given as follows:

$$
\left[\mathbb{M}_{1}\right]=\left(\begin{array}{cccccccc}
1 & 1 & 1 & 1 & -c_{2} & -s_{2} & -c h_{2} & -s h_{2} \\
3 & 2 & 1 & 0 & \gamma_{2} s_{2} & -\gamma_{2} c_{2} & -\gamma_{2} s h_{2} & -\gamma_{2} c h_{2} \\
6 D & 2 D & 0 & 0 & \gamma_{2}^{2} c_{2} & \gamma_{2}^{2} s_{2} & -\gamma_{2}^{2} c h_{2} & -\gamma_{2}^{2} s h_{2} \\
6 D & 0 & 0 & 0 & -\gamma_{2}^{3} s_{2} & \gamma_{2}^{3} c_{2} & -\gamma_{2}^{3} s h_{2} & -\gamma_{2}^{3} c h_{2} \\
0 & 0 & 0 & 1 & 0 & 0 & 0 & 0 \\
0 & 0 & 1 & 0 & 0 & 0 & 0 & 0 \\
0 & 0 & 0 & 0 & -\alpha_{2}^{2} c^{2} & -\alpha_{2}^{2} s^{2} & \alpha_{2}^{2} c h^{2} & \alpha_{2}^{2} s h^{2} \\
0 & 0 & 0 & 0 & \alpha_{2}^{3} s^{2} & -\alpha_{2}^{3} c^{2} & \alpha_{2}^{3} s h^{2} & \alpha_{2}^{3} c h^{2}
\end{array}\right)
$$

$\left[\mathbb{M}_{2}\right]=\left(\begin{array}{cccccccc}c_{1} c h_{1} & c_{1} s h_{1} & s_{1} c h_{1} & s_{1} s h_{1} & -c_{2} & -s_{2} & -c h_{2} & -s h_{2} \\ \gamma_{1} d_{1} & \gamma_{1} d_{2} & \gamma_{1} d_{3} & \gamma_{1} d_{4} & \gamma_{2} s_{2} & -\gamma_{2} c_{2} & -\gamma_{2} s h_{2} & -\gamma_{2} c h_{2} \\ -2 D \gamma_{1}^{2} s_{1} s h_{1} & -2 D \gamma_{1}^{2} s_{1} c h_{1} & 2 D \gamma_{1}^{2} c_{1} s h_{1} & 2 D \gamma_{1}^{2} c_{1} c h_{1} & \gamma_{2}^{2} c_{2} & \gamma_{2}^{2} s_{2} & -\gamma_{2}^{2} c h_{2} & -\gamma_{2}^{2} s h_{2} \\ -2 D \gamma_{1}^{3} d_{4} & 2 D \gamma_{1}^{3} d_{3} & 2 D \gamma_{1}^{3} d_{2} & \left.2 D \gamma_{1}^{3} d_{1}\right) & -\gamma_{2}^{3} s_{2} & \gamma_{2}^{3} c_{2} & -\gamma_{2}^{3} s h_{2} & -\gamma_{2}^{3} c h_{2} \\ 1 & 0 & 0 & 0 & 0 & 0 & 0 & 0 \\ 0 & \gamma_{1} & \gamma_{1} & 0 & 0 & 0 & 0 & 0 \\ 0 & 0 & 0 & 0 & -\alpha_{2}^{2} c^{2} & -\alpha_{2}^{2} s^{2} & \alpha_{2}^{2} c h^{2} & \alpha_{2}^{2} s h^{2} \\ 0 & 0 & 0 & 0 & \alpha_{2}^{3} s^{2} & -\alpha_{2}^{3} c^{2} & \alpha_{2}^{3} s h^{2} & \alpha_{2}^{3} c h^{2}\end{array}\right)$

where:

$c_{i}=\cos \gamma_{i}, \quad s_{i}=\sin \gamma_{i}, \quad c h_{i}=\cosh \gamma_{i}, \quad s h_{i}=\sinh \gamma_{i}$,

$c^{2}=\cos \left(\alpha_{2}+\gamma_{2}\right), \quad s^{2}=\sin \left(\alpha_{2}+\gamma_{2}\right), \quad c h^{2}=\cosh \left(\alpha_{2}+\gamma_{2}\right), \quad s h^{2}=\sinh \left(\alpha_{2}+\gamma_{2}\right)$

$d_{1}=c_{1} s h_{1}-s_{1} c h_{1}, \quad d_{2}=c_{1} c h_{1}-s_{1} s h_{1}, \quad d_{3}=c_{1} c h_{1}+s_{1} s h_{1}, \quad d_{4}=s_{1} c h_{1}+c_{1} s h_{1}$, $\gamma_{1}=\alpha_{1} / \sqrt{2}, \quad \gamma_{2}=l \alpha_{1}$.

\section{References}

[1] Aßmus, M., Naumenko, K., \& Altenbach, H. (2017). Mechanical behaviour of photovoltaic composite structures: Influence of geometric dimensions and material properties on the eigenfrequencies of mechanical vibrations. Composites Communications, 6, 59-62.

[2] Qin, Y., Wang, X., \& Wang, Z.L. (2008). Microfibre-nanowire hybrid structure for energy scavenging. Nature, 451(7180):809.

[3] Schulze, S.H., Pander, M., Naumenko, K., \& Altenbach, H. (2012). Analysis of laminated glass beams for photovoltaic applications. Int. J. Solids Struct., 49(15), 2027-2036.

[4] Martin, T.P., Layman, C.N., Moore, K.M., \& Orris, G.J. (2012). Elastic shells with high-contrast material properties as acoustic metamaterial components. Physical Review B, 85(16), 161103.

[5] Brunet, T., Leng, J., \& Mondain-Monval, O. (2013). Soft acoustic metamaterials. Science, 342(6156), 323-324.

[6] Rus, D., \& Tolley, M.T. (2015). Design, fabrication and control of soft robots. Nature, 521(7553), 467-475. 
[7] Majidi, C. (2014). Soft robotics: a perspective-current trends and prospects for the future. Soft Robot, 1(1), 5-11.

[8] Manna, S., \& Anjali, T.C. (2020). Rayleigh type wave dispersion in an incompressible functionally graded orthotropic half-space loaded by a thin fluid-saturated aeolotropic porous layer. Applied Mathematical Modelling, 83, July, 590-613.

[9] Kudaibergenov, A., Nobili, A., \& Prikazchikova L. (2016). On low-frequency vibrations of a composite string with contrast properties for energy scavenging fabric devices. J. Mech. Mater. Struct., 11, 3, 231-243.

[10] Kaplunov, J., Prikazchikov, D., \& Sergushova O. (2016). Multi-parametric analysis of the lowest natural frequencies of strongly inhomogeneous elastic rods. J. Sound Vib., 366, 264-276.

[11] Kaplunov, J., Prikazchikov, D., Prikazchikova, L.A., \& Sergushova O. (2019). The lowest vibration spectra of multi-component structures with contrast material properties. J. Sound Vib., 445, 132-147.

[12] Şahin, O. (2019). The effect of boundary conditions on the lowest vibration modes of strongly inhomogeneous beams. Journal of Mechanics of Materials and Structures, 14(4), 569-585.

[13] Şahin, O., Erbaş, B., Kaplunov, J., \& Savšek T. (2019). The lowest vibration modes of an elastic beam composed of alternating stiff and soft components. Archive of Applied Mechanics, 1-14, DOI: 10.1007/s00419-019-01612-2.

[14] Hetenyi, M. (1955). Beams on Elastic Foundation. 4th printing. Ann Arbor, Michigan: University of Michigan Press.

[15] Mead, D.J. (1970). Free wave propagation in periodically supported, infinite beams. Journal of Sound and Vibration, 11(2), 181-197.

[16] Yu, D., Wen J., Shen H., Xiao Y., Wen X. (2012). Propagation of flexural wave in periodic beam on elastic foundations. Physics Letters A, 376(4), 626-630.

[17] Zhou, D. (1993). A general solution to vibrations of beams on variable Winkler elastic foundation. Computers and Structures, 47(1), 83-90.

[18] Doyle, P.F., \& Pavlovic M.N. (1982). Vibration of beams on partial elastic foundations. Earthquake Engineering and Structural Dynamics, 10(5), 663-674.

[19] Wang, J. (1991). Vibration of stepped beams on elastic foundations. Journal of Sound and Vibration, 149(2), 315-322.

[20] Coşkun, I., \& Engin H. (1999). Non-linear vibrations of a beam on an elastic foundation. Journal of Sound and Vibration, 223, 335-354.

[21] Coşkun, I. (2000). Non-linear vibrations of a beam resting on a tensionless Winkler foundation. Journal of Sound and Vibration, 236(3), 401-411.

[22] Karahan, M.F., \& Pakdemirli, M. (2017). Vibration analysis of a beam on a nonlinear elastic foundation. Structural Engineering and Mechanics, 62(2), 171-178.

[23] Kural, S., \& Özkaya, E. (2017). Size-dependent vibrations of a micro beam conveying fluid and resting on an elastic foundation. Journal of Vibration and Control, 23(7), 1106-1114.

[24] Mahmoudpour, E., Hosseini-Hashemi, S.H., \& Faghidian, S.A. (2018). Nonlinear vibration analysis of FG nano-beams resting on elastic foundation in thermal environment using stress-driven nonlocal integral model. Applied Mathematical Modelling, 57, 302-315.

[25] Elishakoff, I., Ajenjo, A., \& Livshits, D. (2020). Generalization of Eringen's result for random response of a beam on elastic foundation. European Journal of Mechanics-A/Solids, 81, 103931. 